\title{
A study of the Bergman projection in certain Hartogs domains
}

\author{
Christer O. Kiselman
}

Contents:

1. Introduction

2. Worm domains

3. Functions which are locally independent of the second variable

4. The Bergman projection in a disk

5. A domain where the Bergman projection does not preserve smoothness

6. A smoothly bounded domain in a manifold

References

Resumo: Studo de la projekcio de Bergman en kelkaj Hartogs-aj regionoj Oni montras ke la Bergman-a projekcio ne konservas glatecon de funkcioj en kelkaj pseŭdokonveksaj malfermitaj aroj en la spaco de du kompleksaj variabloj.

Abstract: We show that the Bergman projection does not preserve smoothness of functions in some pseudoconvex domains in the space of two complex variables.

\section{Introduction}

The purpose of this paper is to present two examples of domains where the Bergman projection does not preserve smoothness.

In the space $L^{2}(\Omega)$ of square-integrable functions in an open set $\Omega \subset \mathbf{C}^{n}$, the holomorphic functions form a closed subspace $\mathcal{O}^{2}(\Omega)=L^{2}(\Omega) \cap \mathcal{O}(\Omega)$, the Bergman space. The orthogonal projection onto that subspace is the Bergman projection $P: L^{2}(\Omega) \rightarrow \mathcal{O}^{2}(\Omega)$. If $\Omega$ is bounded, the space $C^{\infty}(\bar{\Omega})$ of functions which are smooth up to the boundary is a subset of $L^{2}(\Omega)$. The question we study is whether $P$ preserves smoothness in the sense that $P\left(C^{\infty}(\bar{\Omega})\right)$ is contained in $C^{\infty}(\bar{\Omega})$.

A Hartogs domain in the space $\mathbf{C}^{2}$ of two complex variables is a domain which contains along with $(z, w)$ also every point $\left(z, w^{\prime}\right)$ with $\left|w^{\prime}\right|=|w|$. It is said to be a complete Hartogs domain if it contains with $(z, w)$ also $\left(z, w^{\prime}\right)$ for all $w^{\prime}$ with $\left|w^{\prime}\right| \leqslant|w|$. Barrett [1984] has found a smoothly bounded Hartogs domain in $\mathbf{C}^{2}$ where the Bergman projection does not preserve smoothness. His domain is not pseudoconvex. It is interesting to compare this with the result of Boas \& Straube [1989] which shows that in a complete Hartogs domain (whether pseudoconvex or not) the Bergman projection maps $C^{\infty}(\bar{\Omega})$ into itself.

Here we will show that in a suitable pseudoconvex Hartogs domain in $\mathbf{C}^{2}$, whose boundary is not smooth, the Bergman projection does not preserve $C^{\infty}(\bar{\Omega})$. This gives rise to a smoothly bounded pseudoconvex open subset of a two-dimensional manifold $\mathbf{C} \times((\mathbf{C} \backslash\{0\}) / \sim)$ where the Bergman projection does not preserve smoothness. 
Theorem 1. Let

$$
\Omega=\left\{(z, w) \in \mathbf{C}^{2} ; a<\log |w|<\left.b \&|z-| w\right|^{i} \mid<1\right\}
$$

where $b-a=m \pi$ for some $m=1,2,3, \ldots$. It is a bounded pseudoconvex domain and its boundary is $C^{\infty}$ except at the points which satisfy $|w|=e^{a},\left|z-e^{i a}\right|=1$ or $|w|=e^{b},\left|z-e^{i b}\right|=1$. Then there is a function $f \in C^{\infty}(\bar{\Omega})$ such that its Bergman projection $P f$ is not Hölder continuous in $\bar{\Omega}$.

Now let $\theta$ be a positive number and let $X_{\theta}$ denote the compact complex manifold obtained from $\mathbf{C} \backslash\{0\}$ by identifying $w$ and $e^{\theta} w$. If $\theta$ is an integer multiple of $2 \pi$, then

$$
Y=\left\{(z, w) \in \mathbf{C} \times X_{\theta} ;\left.|z-| w\right|^{i} \mid<1\right\}
$$

is a pseudoconvex domain in $\mathbf{C} \times X_{\theta}$ with real-analytic boundary. We can define in a natural way a Bergman space $\mathcal{O}^{2}(Y)$ consisting of differential forms of type $(2,0)$ and a Bergman projection $P: L^{2}(Y) \rightarrow \mathcal{O}^{2}(Y)$ (see section 6).

Theorem 2. Let $Y$ be the smoothly bounded pseudoconvex domain just defined, with $\theta=2 \pi m$ for some $m=2,3,4, \ldots$. Then the Bergman projection does not map the forms with coefficients in $C^{\infty}(\bar{Y})$ into the space of continuous forms on $\bar{Y}$.

Domains with similar properties were studied by Barrett [1986].

The structure of the proof of Theorem 1 is as follows. On a subspace $C^{+}(\Omega)$ of $L^{2}(\Omega)$ which contains all Hölder-continuous functions in $\bar{\Omega}$ we construct a linear functional $T$ whose values are obtained as holomorphic extensions of inner products $\left\langle f, g_{\alpha}\right\rangle$ with certain elements $g_{\alpha}$ of the Bergman space. More precisely, for a fixed $f \in C^{+}(\Omega)$ we define a holomorphic function $\Phi(\alpha)=\left\langle f, g_{\alpha}\right\rangle$ of a complex variable $\alpha$ in the half-plane $\operatorname{Re} \alpha>-1$. Then $T(f)$ is the point value $T(f)=\Phi(-2)$ of the extension. The functional $T$ has the following properties.

(a) If both $f$ and $P f$ belong to $C^{+}(\Omega)$, then $T(P f)=T(f)$, for $f-P f$ is orthogonal to $\mathcal{O}^{2}(\Omega)$, in particular to the $g_{\alpha}$.

(b) $T(f)=0$ if $f$ is in $C^{+}(\Omega)$ and holomorphic.

(c) $T$ is not identically zero on $C^{+}(\Omega)$; more precisely, $C^{+}(\Omega)$ contains $C^{\infty}(\bar{\Omega})$ and

$T$ is not zero on the latter space.

To finish the proof we only have to take an $f \in C^{\infty}(\bar{\Omega})$ with $T(f) \neq 0$. If $P f$ did belong to $C^{+}(\Omega)$, then it would follow from (a) that $T(P f)=T(f) \neq 0$, contradicting (b). For details see section 5.

The logical setup of the proof of Theorem 2 is similar, but the functional we use is different. See section 6 .

I am grateful to John Erik Fornæss for comments on an early version of this paper. 


\section{Worm domains}

Diederich \& Fornæss [1977] defined a one-parameter family of pseudoconvex domains which have become known as worm domains. Each of them is a domain $\Omega$ in the space of two complex variables $(z, w)$ whose intersection with any complex subspace $w=$ constant is a disk in the $z$-plane with center at the point $|w|^{i}=\exp (i \log |w|)$ and radius $R(\log |w|) \leqslant 1$. If $R(\log |w|)=1$, then the point $(0, w)$ is on the boundary of $\Omega$. Let us agree to call any domain of the form

$$
\Omega=\left\{(z, w) \in \mathbf{C}^{2} ;\left.|z-| w\right|^{i} \mid<R(\log |w|)\right\}
$$

a worm domain. In particular they are Hartogs domains and can be easily visualized in the space of three real variables $(\operatorname{Re} z, \operatorname{Im} z, \log |w|)$. We shall give a condition on the radius $R$ which implies pseudoconvexity. It will be convenient to consider $\psi=1-R^{2}$. We allow $\psi<0$, i. e., $R>1$. Where $\psi>1$ there are no corresponding points in $\Omega$.

Let us say that a function $\psi$ defined on some interval of $\mathbf{R}$ is trigonometrically convex if $\psi^{\prime \prime}+\psi \geqslant 0$ in the distribution sense. This amounts to $\psi(\arg \zeta)|\zeta|$ being convex (or, equivalently, subharmonic) in some sector in the complex plane. The easiest examples are the convex nonnegative functions. Other examples are $a$ sin and $a \sin ^{+}=a \max (\sin , 0), a>0$.

The following result is due to Diederich \& Fornæss [1977], pages 277-279, for a particular choice of the function $\psi$.

Proposition 2.1. Let $-\infty \leqslant a<b \leqslant+\infty$ and let $\psi$ be a trigonometrically convex function on the open interval $] a, b[$. Then

$$
\Omega=\left\{(z, w) \in \mathbf{C}^{2} ; a<\log |w|<\left.\left.b \&|z-| w\right|^{i}\right|^{2}+\psi(\log |w|)<1\right\}
$$

is pseudoconvex. If $\psi$ is of class $C^{k}$ for some $k=1,2,3, \ldots, \infty$, then $\partial \Omega$ is of class $C^{k}$ except possibly where $\psi=1$ or $|w|=e^{a}$ or $|w|=e^{b}$; if in addition $\psi(a+)>1$, $\psi(b-)>1$ and $\psi^{\prime} \neq 0$ when $\psi=1$, then $\partial \Omega$ is of class $C^{k}$ everywhere.

Proof. Locally we can introduce $\zeta=w^{-i}$ as a new variable; then $\log |w|=-\arg \zeta$ and $\arg w=\log |\zeta|$. The condition for a point $(z, w)$ with $a<\log |w|<b$ to belong to $\Omega$, in addition to the obvious $a<-\arg \zeta<b$, becomes

$$
|z|^{2}-2 \operatorname{Re}\left(z e^{i \arg \zeta}\right)+\psi(-\arg \zeta)<0
$$

We can multiply this by $|\zeta|$ to get the equivalent condition

$$
|z|^{2}|\zeta|-2 \operatorname{Re}(z \zeta)+\psi(-\arg \zeta)|\zeta|<0
$$

Here all terms are plurisubharmonic in $(z, \zeta)$ : the first is the exponential of the plurisubharmonic function $2 \log |z|+\log |\zeta|$, the second is the real part of a holomorphic function, and the third is convex in $\zeta$ by hypothesis. Therefore the set where this function is negative is pseudoconvex. 
If $\psi(a+)>1$ and $\psi(b-)>1$ there can be a question about the smoothness of the boundary only where the radius of the disk in the $z$-plane is zero, i. e., where $\psi=1$. Consider the domain

$$
\Omega^{\prime}=\left\{(z, w) \in \mathbf{C}^{2} ; a<\log |w|<b \&|z-1|^{2}+\psi(\log |w|)<1\right\}
$$

which is diffeomorphic to $\Omega$ under the diffeomorphism $(z, w) \mapsto\left(z|w|^{-i}, w\right)$ (defined in a neighborhood of $\bar{\Omega}$ ). If $\psi$ satisfies the condition mentioned at the points where it takes the value 1 , then the boundary of $\Omega^{\prime}$ near the points where the radius is zero is given by the equation $\psi(\log |w|)=1-|z-1|^{2}$. We can write this as $\log |w|=$ $\psi^{-1}\left(1-|z-1|^{2}\right)$, where $z \mapsto 1-|z-1|^{2}$ is $C^{\infty}$, and where the inverse $\psi^{-1}$ is as smooth as $\psi$. This proves the proposition.

The simplest choice is $a=-\infty, b=+\infty, R=1$ identically; this defines an unbounded pseudoconvex domain $\Omega_{0}$ whose boundary is $C^{\infty}$ except where $w=0$. Another choice is $-\infty<a<b<+\infty, R=1$; then $\Omega$ is bounded and its boundary is smooth except where $|w|=e^{a},\left|z-e^{i a}\right|=1$ or $|w|=e^{b},\left|z-e^{i b}\right|=1$. The boundary of these domains contains a piece of the analytic manifold $z=0$.

It is easy to define smoothly bounded domains using Proposition 2.1: just take a $C^{\infty}$ convex function $\psi$ on $[a, b]$ which is zero on some interval $\left[a_{0}, b_{0}\right]$, positive outside this interval and satisfies $\psi(a)>1, \psi(b)>1$. Since $\psi$ is nonnegative and convex, it is trigonometrically convex, and $\Omega$ is contained in the set where $\psi<1$. There exist two points $a_{1}<b_{1}$ such that $\psi\left(a_{1}\right)=\psi\left(b_{1}\right)=1$; obviously $\psi^{\prime}\left(a_{1}\right)<0, \psi^{\prime}\left(b_{1}\right)>0$. Therefore $\partial \Omega$ is $C^{\infty}$.

We now ask whether the domains in Proposition 2.1 can be defined by a global plurisubharmonic function. Diederich \& Fornæss [1977] proved (pages 284-285) that their worm domains do not possess a plurisubharmonic defining function of class $C^{3}$. We shall prove a somewhat stronger result here.

Proposition 2.2. Let $\Omega$ be as in Proposition 2.1 with $\psi \geqslant 0$ everywhere and $\psi=0$ (i. e., $R=1$ ) on some interval $\left[a_{0}, b_{0}\right]$ of positive length. Denote the distance to the boundary in $\Omega$ by $d_{\Omega}$. Then there is no function $f \in P S H(\Omega)$ satisfying $-A d_{\Omega} \leqslant$ $f \leqslant-B d_{\Omega}$ in $\Omega$ with positive constants $A$ and $B$. In particular there cannot exist a global plurisubharmonic defining function for $\Omega$ which is Lipschitz continuous. $A$ little more generally, if $\varepsilon>\pi /\left(b_{0}-a_{0}+\pi\right)$, there cannot exist a function $f \in \operatorname{PSH}(\Omega)$ satisfying $-A d_{\Omega}^{\varepsilon} \leqslant f \leqslant-B d_{\Omega}^{\varepsilon}$ in $\Omega$ with positive $A$ and $B$.

Proof. Suppose there is a plurisubharmonic function $f$ with $-A d_{\Omega}^{\varepsilon} \leqslant f \leqslant-B d_{\Omega}^{\varepsilon}$. Define first

$$
f_{0}(z, w)=\sup _{\left|w^{\prime}\right|=|w|} f\left(z, w^{\prime}\right), \quad(z, w) \in \Omega .
$$

Then $f_{0}$ is a function of $(z,|w|)$, it is plurisubharmonic in $\Omega$ and satisfies the same inequality as $f$ there, for $d_{\Omega}(z, w)=d_{\Omega}\left(z, w^{\prime}\right)$ if $|w|=\left|w^{\prime}\right|$. Next define

$$
F(z)=\inf _{w} f_{0}(z, w)
$$

In view of the minimum principle for plurisubharmonic functions (Kiselman [1978], Theorem 2.2), $F$ is subharmonic on some Riemann surface in $z$. We may suppose 
without loss of generality that $a_{0}<0<b_{0}$, so that this Riemann surface contains the half-disk

$$
U=\{z \in \mathbf{C} ;|z|<r \& \operatorname{Re} z>0\}
$$

for some positive $r$. If $-A d_{\Omega} \leqslant f \leqslant-B d_{\Omega}$, then $F$ satisfies $-A_{1}|z| \leqslant F(z) \leqslant-B_{1}|z|$ in $U$ for some new positive constants $A_{1}$ and $B_{1}$, and $F$ has a continuous extension to the closure $\bar{U}$. We introduce an auxiliary function

$$
G(z)=|z|\left(1-\frac{1}{2} \log \frac{|z|}{r} \cos \arg z\right) .
$$

We note that $G$ is subharmonic in $U$, has a continuous extension to the closure of $U$ and coincides with $|z|$ on the boundary of $U$. Consider $F+B_{1} G$. This function is $\leqslant 0$ on the boundary of $U$. But at the point $z=r \exp \left(-2 A_{1} / B_{1}\right) \in U$ it assumes a positive value: this contradicts the maximum principle for subharmonic functions and completes the proof in the case $\varepsilon=1$.

For the general case we define $F_{\varepsilon}(z)=F\left(z^{1 / \varepsilon}\right)$ in $U$ for a sufficiently small positive $r$. This is possible if $\arg i^{1 / \varepsilon}<b_{0}+\pi / 2$ and $\arg (-i)^{1 / \varepsilon}>a_{0}-\pi / 2$. It is however no restriction to assume that $b_{0}=-a_{0}$; then both conditions become $\pi / \varepsilon<b_{0}-a_{0}+\pi$. The function $F_{\varepsilon}$ has a continuous extension to $\bar{U}$ and satisfies $-A_{1}|z| \leqslant F_{\varepsilon}(z) \leqslant-B_{1}|z|$. Thus the same argument applies, completing the proof of Proposition 2.2.

It is interesting to note in this context the result due to Bonami \& Charpentier [1988]: the Bergman projection preserves the Sobolev class $W^{1 / 2}(\Omega)$ in a domain with boundary of class $C^{3}$ and admitting a global plurisubharmonic defining function.

\section{Functions which are locally independent of the second variable}

In the sets $\Omega$ as defined in the last section the functions which are locally independent of the second variable $w$ play an important role.

Proposition 3.1. Let $\Omega$ be a Hartogs domain in $\mathbf{C}^{2}$. If $f \in L^{2}(\Omega)$ is of the form $f(z, w)=w^{k} F(z,|w|)$ for some $k \in \mathbf{Z}$, then $w^{-k} P f$ is locally independent of $w$.

Proof. If $a$ is a complex number of modulus one, the mapping $f \mapsto f \circ T_{a}$ defined by $T_{a}(z, w)=(z, a w)$ is an isometry $T_{a}^{*}$ of $L^{2}(\Omega)$ which moreover preserves the subspace $\mathcal{O}^{2}(\Omega)$. This implies that $T_{a}^{*}$ and $P$ commute. Now if $f(z, w) / w^{k}$ is a function of $(z,|w|)$ (for $w \neq 0$ ), we have $T_{a}^{*} f=a^{k} f$ for all numbers $a$ of modulus one, so that $T_{a}^{*} P f=P T_{a}^{*} f=P\left(a^{k} f\right)=a^{k} P f$. Therefore $H(z, w)=w^{-k} P f(z, w)$ satisfies $T_{a}^{*} H=H$ : it is invariant under rotation in the $w$-space and so must be a function of $(z,|w|)$. Since $H$ is holomorphic, this implies that it is locally independent of $w$.

Proposition 3.2. Let $\Omega$ be defined as in Proposition 2.1 with a nonnegative $\psi$ such that $\psi^{-1}(0)$ is an interval. Assume $f \in C(\Omega)$ is locally independent of $w$ and $\lim f(z, w)=f_{0}(w)$ exists as $z \rightarrow 0,(z, w) \in \Omega$, for every $w$ such that $(0, w) \in \partial \Omega$. Then $f_{0}$ is constant.

Proof. In particular $f_{0}$ is equal to the limit

$$
f_{0}(w)=\lim _{t \rightarrow 0} f(t z, w)
$$


where $t>0$ and $z$ is chosen so that $(t z, w)$ is in $\Omega$ for all $t$ with $0<t<\varepsilon$ for some positive $\varepsilon$. The same $z$ can serve for all $w$ near the original point. Therefore $f_{0}$ is locally constant. Now the set of $w$ we are considering is connected: it is the annulus $e^{a_{0}} \leqslant|w| \leqslant e^{b_{0}}$ where $\left[a_{0}, b_{0}\right]=\psi^{-1}(0)$. So locally constant is the same thing as being constant.

It will be important for us to have a supply of functions which are holomorphic and locally independent of $w$. Let $\Omega_{0}$ be the set defined by (2.1) with the special choices $a=-\infty, b=+\infty$, and $\psi=0$. We define a function in $\Omega_{0}$ which we call simply $\log z$, by first defining it for $w=1$ in the disk

$$
D_{1}=\{z \in \mathbf{C} ;|z-1|<1\}
$$

as the branch of the $\operatorname{logarithm}$ with $\log 1=0$. Then we continue it analytically from $D_{1} \times\{1\} \subset \Omega_{0}$ to all of $\Omega_{0}$. As in Diederich \& Fornæss [1977], $\S 2$, we can now define, for any complex number $\alpha$, a function

$$
g_{\alpha}(z, w)=\exp (\alpha \log z)
$$

of $(z, w)$ which we may also denote by $z^{\alpha}$, at least if we keep in mind that it is not globally a function of $z$ when $\alpha \in \mathbf{C} \backslash \mathbf{Z}$.

\section{The Bergman projection in a disk}

Here we shall recall some formulas for the Bergman projection in a disk. In the unit disk $D=\{z \in \mathbf{C} ;|z|<1\}$ we have an explicit expression for the Bergman projection $B: L^{2}(D) \rightarrow \mathcal{O}^{2}(D):$

$$
B f(a)=\int_{D} f(z) \frac{1}{\pi(1-\bar{z} a)^{2}} d \lambda(z), \quad a \in D
$$

see e. g. Krantz [1982:50]. Here $\lambda$ denotes Lebesgue measure in C. We change variables in (4.1) to get the projection in the disk $D_{r}=\{z \in \mathbf{C} ;|z-1|<r\}$ and write $B: L^{2}\left(D_{r}\right) \rightarrow \mathcal{O}^{2}\left(D_{r}\right)$ for the Bergman projection there also. This gives

$$
B f(a)=\int_{D_{r}} f(z) \overline{\varphi_{a}(z)} d \lambda(z), \quad a \in D_{r}
$$

where

$$
\varphi_{a}(z)=\frac{\left(1-a^{*}\right)^{2}}{\pi r^{2}\left(z-a^{*}\right)^{2}}, \quad a, z \in D_{r},
$$

and $a^{*}$ is the point defined by reflection in $\partial D_{r}$, in other words $a^{*}$ is determined by $(\bar{a}-1)\left(a^{*}-1\right)=r^{2}$. If $0<r<1$, we can in particular choose $a^{*}=0, a=1-r^{2}$ :

$$
\int_{D_{r}} \frac{f(z)}{\bar{z}^{2}} d \lambda(z)=\pi r^{2} B f\left(1-r^{2}\right), \quad 0<r<1 .
$$

In a suitable sense (4.3) holds also for $r=1$ if $f$ is Hölder continuous in $\bar{D}_{1}$. The interpretation of the integral is then elementary if $f(0)=0$, and one can prove that

$$
\int_{D_{1}} \frac{f(z)}{\bar{z}^{2}} d \lambda(z)=\pi B f(0)
$$


by passing to the limit in (4.2). Here we let $B f$ denote also the continuous extension to the closure of $D_{1}$. Next we write $f(z)=f(0)+(f(z)-f(0))$ and consider the case when $f$ is identically one. Then we note that, in view of the mean value property for antiholomorphic functions,

$$
\int_{D_{r}} \bar{z}^{\alpha} d \lambda(z)=\pi r^{2}, \quad 0<r \leqslant 1, \quad \operatorname{Re} \alpha>-2
$$

We simply extend this function of $(r, \alpha)$ by continuity from $\operatorname{Re} \alpha>-2$ to $\operatorname{Re} \alpha \geqslant-2$. This is the interpretation of (4.3) for $r=1$ which we shall use.

Note that we do not define (4.3) for $r=1$ as a principal value by cutting out a disk around the origin: that will give a different value. In fact, it can be proved that

$$
\lim _{\varepsilon \rightarrow 0} \int_{z \in D_{1},|z|>\varepsilon} \frac{1}{\bar{z}^{2}} d \lambda(z)=\frac{\pi}{2}
$$

\section{A domain where the Bergman projection does not preserve smoothness}

Every holomorphic function in a Hartogs domain $\Omega$ has a Laurent expansion

$$
h(z, w)=\sum_{j \in \mathbf{Z}} h_{j}(z,|w|) w^{j}
$$

where $h_{j} \in \mathcal{O}(\Omega)$. It turns out that in the worm domains (2.1) the functions which are homogeneous of degree -1 exhibit a resonance phenomenon which we will exploit. (It will give rise to property (b) of the introduction, and is made explicit in formula (5.4) below.) We shall therefore calculate the scalar product $\langle f / w, g / w\rangle$ where $f$ and $g$ are two functions in $L^{2}(\Omega)$ satisfying $f(z, w)=f(z,|w|), g(z, w)=g(z,|w|)$, but we shall not suppose that they are holomorphic. Introducing polar coordinates for $w$ with $|w|=r=e^{t}$ we get

$$
\begin{gathered}
\langle f / w, g / w\rangle=\iint_{\Omega} \frac{f(z, w)}{w} \frac{\overline{g(z, w)}}{\bar{w}} d \lambda(z) d \lambda(w)= \\
=2 \pi \iint f(z, r) \overline{g(z, r)} d \lambda(z) d r / r=2 \pi \iiint_{\left|z-e^{i t}\right|<R(t)} f\left(z, e^{t}\right) \overline{g\left(z, e^{t}\right)} d \lambda(z) d t .
\end{gathered}
$$

We now take $z^{\prime}=z e^{-i t}$ as a new variable: the transformation $(z, w) \mapsto\left(z^{\prime}, w\right)$ preserves volumes and we get

$$
\langle f / w, g / w\rangle=2 \pi \iint_{\left|z^{\prime}-1\right|<R(t)} f\left(e^{i t} z^{\prime}, e^{t}\right) \overline{g\left(e^{i t} z^{\prime}, e^{t}\right)} d \lambda\left(z^{\prime}\right) d t .
$$


If $\Omega$ is bounded and defined by a nonnegative function $\psi$, and if $\operatorname{Re} \alpha>-1$, we can in particular choose $g(z, w)=z^{\bar{\alpha}}$ and define

$$
\begin{aligned}
T_{\alpha}(f / w) & =\langle f / w, g / w\rangle=2 \pi \iint_{|z-1|<R(t)} f\left(e^{i t} z, e^{t}\right) \overline{g\left(e^{i t} z, e^{t}\right)} d \lambda(z) d t= \\
& =2 \pi \iint_{|z-1|<R(t)} f\left(e^{i t} z, e^{t}\right) e^{-i \alpha t} \bar{z}^{\alpha} d \lambda(z) d t .
\end{aligned}
$$

Moreover

$$
T_{\alpha}(f / w)=T_{\alpha}(P(f / w))
$$

for these $\alpha$, since $(z, w) \mapsto z^{\bar{\alpha}} / w$ belongs to $\mathcal{O}^{2}(\Omega)$ and $f / w-P(f / w)$ is orthogonal to that space. Note that, by Proposition 3.1, the function $P(f / w)$ is of the form $h(z,|w|) / w$ so that the scalar product $\langle P(f / w), g / w\rangle$ is given by the same formulas.

The equation (5.2) will of course be preserved under analytic continuation in the parameter $\alpha$. For example, if both $f$ and $P(f / w)$ happen to be bounded, we can extend the equation to the half-plane $\operatorname{Re} \alpha>-2$.

Now if $f$ is measurable and satisfies an estimate $|f(z, w)| \leqslant C|z|^{\varepsilon}$ with $\varepsilon>0$, then $T_{\alpha}(f / w)$ is well defined by (5.1) for $\operatorname{Re} \alpha>-2-\varepsilon$.

If $f$ is continuous on the closure of $\Omega$ and independent of $z$, we may extend $f$ to all of $\mathbf{C}^{2}$ and write $f(0, w)$ for the value of $f$ at any of the points $(z, w) \in \bar{\Omega}$ (even though the corresponding point $(0, w)$ need not belong to $\bar{\Omega})$. In this case we can easily extend $T_{\alpha}(f / w)$ :

$$
T_{\alpha}(f / w)=2 \pi \iint_{|z-1|<R(t)} f\left(0, e^{t}\right) e^{-i \alpha t} \bar{z}^{\alpha} d \lambda(z) d t=2 \pi^{2} \int R(t)^{2} f\left(0, e^{t}\right) e^{-i \alpha t} d t,
$$

for in view of the mean value property for antiholomorphic functions, the integral with respect to $z$ is equal to the area $\pi R(t)^{2}$ times the value at the center of the disk. The last integral has a sense for all $\alpha \in \mathbf{C}$.

If $f$ is Hölder continuous with exponent $\varepsilon$ in $\bar{\Omega}$ we can extend $T_{\alpha}(f / w)$ to all $\alpha$ satisfying $\operatorname{Re} \alpha>-2-\varepsilon$ by combining these two ideas: we shall assume that $f$ is defined in all of $\mathbf{C}^{2}$ and write $f(z, w)=f(0, w)+(f(z, w)-f(0, w))$. We now apply the functional $T_{\alpha}$ separately to the two terms. We see that if $\operatorname{Re} \alpha>-2$, then $T_{\alpha}(f / w)$ can be written as

$$
2 \pi^{2} \int R(t)^{2} f\left(0, e^{t}\right) e^{-i \alpha t} d t+2 \pi \iint\left(f\left(e^{i t} z, e^{t}\right)-f\left(0, e^{t}\right)\right) e^{-i \alpha t} \bar{z}^{\alpha} d \lambda(z) d t .
$$

Now the first term has a sense for all $\alpha$, and the second is defined as an integral for all $\alpha$ in the half-plane $\operatorname{Re} \alpha>-2-\varepsilon$. The extension so obtained seemingly depends on the values of $f(0, w)$ also for $w$ outside the annuli where $f(0, w)$ is defined originally (these annuli are defined by the condition that $\log |w|$ belong to $\psi^{-1}(0)$ which is a union of intervals), but it actually does not, since $T_{\alpha}(f / w)$ is a function of $f$ restricted to $\Omega$ for $\alpha$ satisfying $\operatorname{Re} \alpha>-2$, and then this must be true also of its holomorphic extension.

Of course we do not need Hölder continuity everywhere in $\bar{\Omega}$, so we can summarize the discussion in the following definition: 
Definition 5.1. For a worm domain $\Omega$ with $R \leqslant 1$ we let $C^{+}(\Omega)$ denote the space of all bounded measurable functions $f$ defined in $\Omega$ which satisfy an estimate

$$
\left|f(z, w)-f_{0}(w)\right| \leqslant C|z|^{\varepsilon}, \quad(z, w) \in \Omega,
$$

for a suitable measurable function $f_{0}$ and some constants $C$ and $\varepsilon>0$. We define

$$
T_{-2}(f / w)=\lim _{\substack{\alpha \rightarrow-2 \\ \operatorname{Re} \alpha>-2}} T_{\alpha}(M(f) / w), \quad f \in C^{+}(\Omega),
$$

where $T_{\alpha}$ is given by (5.1) for $\operatorname{Re} \alpha>-2$, and where $M(f)$ denotes the mean value of $f(z, t w)$ over the circle $|t|=1$.

The function $f_{0}$ can be thought of as an extension of $w \mapsto f(0, w)$; it is not unique since $(z, w) \in \bar{\Omega}, R(\log |w|)<1$ implies $(0, w) \notin \bar{\Omega}$.

We can now use the formulas for the Bergman projection in a disk (see section 4) to express $T_{-2}$. It will be enough to consider functions $f \in C^{+}(\Omega)$ which are are functions of $(z,|w|)$ so that $M(f)=f$. If such an $f$ is holomorphic in $z$ for fixed $w$, we get writing $I$ for the open set where $R>0, \psi<1$, and $J=\psi^{-1}(0)$ for the set where $R=1, \psi=0$,

$$
\begin{aligned}
T_{-2}(f / w) & =2 \pi^{2} \int R(t)^{2} f\left(e^{i t}\left(1-R(t)^{2}\right), e^{t}\right) e^{2 i t} d t= \\
& =2 \pi^{2} \int_{J} f\left(0, e^{t}\right) e^{2 i t} d t+2 \pi^{2} \int_{I \backslash J} R(t)^{2} f\left(\psi(t) e^{i t}, e^{t}\right) e^{2 i t} d t .
\end{aligned}
$$

These formulas clearly use only values of $f$ in $\bar{\Omega}$. It is by no means obvious how to estimate the integral over $I \backslash J$. However, in the special case when $R=1$ in the whole interval $] a, b[$ this term vanishes. Then we obtain the following result, which implies Theorem 1 of the introduction:

Theorem 5.2. Let

$$
\Omega=\left\{(z, w) \in \mathbf{C}^{2} ; a<\log |w|<\left.b \&|z-| w\right|^{i} \mid<1\right\},
$$

where $b-a=m \pi$ for some $m=1,2,3, \ldots$. Let $f \in C^{+}(\Omega)$ be holomorphic in $z$ for fixed $w$ and satisfy $f(z, w)=f(z,|w|), \int f\left(0, e^{t}\right) e^{2 i t} d t \neq 0$. Then $P(f / w)$, the Bergman projection of $f / w$, is not in $C^{+}(\Omega)$.

We can easily find $C^{\infty}$ functions $f$ which satisfy the hypotheses of the theorem; for instance $f(z, w)=g(\log |w|)$, where $g \in C^{\infty}(\mathbf{R})$ with support in $[a, b]$ and Fourier transform $\hat{g}$ satisfying $\hat{g}(-2) \neq 0$.

Proof. In view of Proposition 3.1, $P(f / w)=h / w$, where $h$ is a holomorphic function of $(z,|w|)$. If $h$ satisfied an estimate $|h(z, w)-h(0, w)| \leqslant C|z|^{\varepsilon}$, we would get $T_{-2}(h / w)=T_{-2}(f / w) \neq 0$ from (5.2). On the other hand, (5.3) yields

$$
T_{-2}(h / w)=2 \pi^{2} \int_{J} h\left(0, e^{t}\right) e^{2 i t} d t=2 \pi^{2} h\left(0, e^{s}\right) \int_{J} e^{2 i t} d t=0,
$$

where we write $h\left(0, e^{s}\right)$ for the common value of $h(0, w)$ for all $w$ such that $(0, w) \in$ $\partial \Omega$; see Proposition 3.2. The last equality follows simply because $J=] a, b[=$ ]$a, a+m \pi[$ so that

$$
\int_{J} e^{2 i t} d t=\int_{a}^{a+m \pi} e^{2 i t} d t=0 .
$$

This contradiction proves the theorem. 


\section{A smoothly bounded domain in a manifold}

Let $\theta$ be a positive number and let $X_{\theta}$ denote the compact complex manifold obtained from $\mathbf{C} \backslash\{0\}$ by identifying $w$ and $e^{\theta} w$. The differential form $d w / w$ is well defined on $X_{\theta}$. We shall let $q: \mathbf{C} \times(\mathbf{C} \backslash\{0\}) \rightarrow \mathbf{C} \times X_{\theta}$ denote the canonical projection which associates with $(z, w)$ its equivalence class in $\mathbf{C} \times X_{\theta}$.

Consider the domain

$$
\Omega=\left\{(z, w) \in \mathbf{C} \times(\mathbf{C} \backslash\{0\}) ;\left.|z-| w\right|^{i} \mid<1\right\},
$$

which is pseudoconvex in view of Proposition 2.1, and its subset

$$
\Omega_{\theta}=\{(z, w) \in \Omega ; 0<\log |w|<\theta\} .
$$

If $\theta=2 \pi m$ is an integer multiple of $2 \pi$, the condition on $(z, w)$ to belong to $\Omega$ does not change if we replace $w$ by $e^{\theta} w$, for in that case $\left|e^{\theta} w\right|^{i}=e^{2 \pi m i}|w|^{i}=|w|^{i}$. Therefore

$$
Y=q(\Omega)=\left\{q(z, w) \in \mathbf{C} \times X_{\theta} ;\left.|z-| w\right|^{i} \mid<1\right\}
$$

is a pseudoconvex domain in $\mathbf{C} \times X_{\theta}$ with real-analytic boundary. We have $q\left(\bar{\Omega}_{\theta}\right)=\bar{Y}$. From now on we shall assume $\theta=2 \pi m$.

We let $L^{2}(Y)$ denote the space of all differential forms of bidegree $(2,0)$ with a representation

$$
F=f(z, w) d z \wedge d w / w
$$

where $f$ is square integrable on bounded subsets of $\Omega$ and satisfies $f\left(z, e^{\theta} w\right)=f(z, w)$. The scalar product is

$$
\langle F, G\rangle=\iint_{\Omega_{\theta}} f(z, w) d z \wedge d w / w \wedge \overline{g(z, w) d z \wedge d w / w}
$$

The Bergman space $\mathcal{O}^{2}(Y)$ is the subspace of $L^{2}(Y)$ with $f$ holomorphic. It is contained in a natural way in the Bergman space $\mathcal{O}^{2}\left(\Omega_{\theta}\right)$ : an element of the latter belongs to the former if it has an extension to all of $\Omega$ which is periodic in the sense that $f\left(z, e^{\theta} w\right)=f(z, w)$. Our result that $T_{-2}(f / w)=0$ for all holomorphic Hölder-continuous $f$ in $\bar{\Omega}_{\theta}$ is therefore true also for $Y$.

The function $g_{\alpha}(z, w)=\exp (\alpha \log z)$ (see the end of section 3 ) is well defined in $Y$ if $\alpha \theta / 2 \pi$ is an integer. For instance, if $\theta=4 \pi$, then $\sqrt{z}$ is well defined in $Y$, although it is not globally a function of $z$, and both $\sqrt{z} d z \wedge d w / w$ and $1 / \sqrt{z} d z \wedge d w / w$ are elements of the Bergman space. But there seems to be no reason why the equation $T_{-2}(P f)=T_{-2}(f)$, which was crucial in the proof of Theorem 5.2, should hold, for this was a consequence of the fact that $T_{-2}$ appeared as a limit of scalar products with functions $z^{\bar{\alpha}}$, and such functions are defined on the manifold $X_{\theta}=X_{2 \pi m}$ only if $\alpha$ is a rational number of the form $\alpha=k / m$ with $k \in \mathbf{Z}$. (Moreover the corresponding form belongs to the Bergman space only if $k>-m$.) This is the reason why we have not been able to use the functional $T_{-2}$ in the present proof. We shall therefore construct another functional $S$ as follows. 
Definition 6.1. Let $\gamma$ denote the curve $[0, \theta] \ni t \mapsto\left(e^{i t}, e^{t}\right) \in \bar{\Omega}_{\theta}$ which traces the centers of the disks in the z-plane. Then $q \circ \gamma$ is a closed curve in $Y$. We let $N(Y)$ denote the set of all functions $f \in C(Y)$ which do not vanish on $q \circ \gamma$. We define a functional $S$ by the change in argument of the number $f(q(z, w))$ along $\gamma$ :

$$
S(f)=\frac{1}{2 \pi i} \underset{\gamma}{\operatorname{var}} \arg f(q(z, w))=\frac{1}{2 \pi i} \underset{q \circ \gamma}{\operatorname{var}} \arg f, \quad f \in N(Y) .
$$

We list a few of the properties of $S$ :

Lemma 6.2. If $f \in N(Y) \cap \mathcal{O}(Y) \cap C(\bar{Y})$ is a function of $(z,|w|)$, then $S(f) \in \mathbf{N}$.

Proof. We define three segments $\gamma_{0}, \gamma_{1}, \gamma_{2}$ as follows:

$$
\gamma_{0}:[0, \theta] \ni t \mapsto\left(0, e^{\theta-t}\right), \quad \gamma_{1}:[0,1] \ni t \mapsto(t, 1), \quad \gamma_{2}:[0,1] \ni t \mapsto\left(1-t, e^{\theta}\right)
$$

They are all contained in the boundary of $\Omega_{\theta}$. Let $\Gamma$ be the closed curve in $\bar{\Omega}_{\theta}$ consisting of $\gamma_{1}+\gamma+\gamma_{2}+\gamma_{0}$. Now clearly

$$
\underset{q \circ \Gamma}{\operatorname{var}} \arg f=\underset{q \circ \gamma}{\operatorname{var}} \arg f
$$

for any $f \in C(\bar{Y})$ which is holomorphic, locally independent of $w$, and nowhere zero on $q \circ \Gamma$. For by Proposition 3.2, $f \circ q$ is constant on $\gamma_{0}$, and the contributions from $\gamma_{1}$ and $\gamma_{2}$ cancel each other, since $q(t, 1)=q\left(t, e^{\theta}\right)$. If $f$ satisfies only the hypotheses of the lemma, we cannot of course guarantee that $f$ is never zero on $q \circ \Gamma$. But $f\left(q \circ \gamma_{0}\right)$ is a point, and $f\left(q \circ \gamma_{1}\right) \cup f\left(q \circ \gamma_{2}\right)$ is a countable union of smooth arcs, hence a set of measure zero. Therefore $f(q \circ \Gamma)$ is the union of $f(q \circ \gamma)$ and a compact set of measure zero, so there are infinitely many complex numbers $a$ of arbitrarily small modulus such that $f-a$ is nowhere zero on $q \circ \Gamma$. If we take any such $a$ with modulus smaller than $\inf _{q \circ \gamma}|f|$, then we know that

$$
S(f)=\frac{1}{2 \pi i} \underset{q \circ \Gamma}{\operatorname{var}} \arg (f-a) .
$$

Now if $f \circ q$ is a function of $(z,|w|)$, then given any sufficiently small positive number $\varepsilon$ (it suffices that $\varepsilon<2 \pi /(\theta+\pi)=1 /(m+1 / 2)$ ), we can write

$$
f(q(z, w))=f(q(z,|w|))=g\left(z^{\varepsilon}\right)
$$

for some function $g$. When $(z, w)$ runs through $\Gamma$, the point $z^{\varepsilon}$ runs through a curve which we call $\Gamma_{\varepsilon}$, and which consists of the segment from 0 to 1 followed by the arc of the unit circle from 1 to $e^{i \varepsilon \theta}$, followed by the segment from that point back to the origin. The function $g$ is continuous on the closure of the sector surrounded by $\Gamma_{\varepsilon}$ and holomorphic inside the curve. And

$$
\underset{q \circ \Gamma}{\operatorname{var}} \arg (f-a)=\underset{\Gamma_{\varepsilon}}{\operatorname{var}} \arg (g-a)=2 \pi i N,
$$

where $N \geqslant 0$ is the number of zeros of $g-a$ inside the curve $\Gamma_{\varepsilon}$, by the usual argument principle. This proves the lemma. 
Lemma 6.3. The set of all forms $F=f d z \wedge d w / w \in \mathcal{O}^{2}(Y)$ with $f \in N(Y)$ is open in $\mathcal{O}^{2}(Y)$ and the functional $S$ restricted to such forms is locally constant for the Bergman topology.

Proof. We know that point evaluations are continuous in the Bergman norm and moreover uniformly so over any compact set. Since the image of $q \circ \gamma$ is compact in $Y$, there is a constant $C$ such that

$$
\sup _{q \circ \gamma}|f| \leqslant C\|F\|, \quad F \in \mathcal{O}^{2}(Y) .
$$

We apply this to $G-F \in \mathcal{O}^{2}(Y)$ :

$$
\sup _{q \circ \gamma}|g-f| \leqslant C\|G-F\| \text {. }
$$

For a fixed $F$ with $f \in N(Y)$ we define $\varepsilon=\inf _{q \circ \gamma}|f| / C>0$. Then $\inf _{q \circ \gamma}|g|>0$ for all $G=g d z \wedge d w / w \in \mathcal{O}^{2}(Y)$ such that $\|G-F\|<\varepsilon$, i. e., we have proved that $g \in N(Y)$. The change in argument for $g$ must be the same as for $f$.

Theorem 6.4. Let $Y$ be the smoothly bounded pseudoconvex subset of $\mathbf{C} \times X_{\theta}$ defined by (6.1), with $\theta=2 \pi m$ for some $m=2,3,4, \ldots$. Then

$$
F=z^{-1 / m} d z \wedge d w / w
$$

is an element of the Bergman space $\mathcal{O}^{2}(Y)$. Let $\varphi \in C^{\infty}(\mathbf{C})$ vanish near 0 , satisfy $0 \leqslant \varphi \leqslant 1$, and be such that

$$
\{z \in \mathbf{C} ;|z|<2 \& \varphi(z) \neq 1\}
$$

has sufficently small Lebesgue measure. Then $\varphi F$ is a smooth form, and its Bergman projection is

$$
P(\varphi F)=h(z, w) d z \wedge d w / w
$$

with a holomorphic function $h$ which cannot be continuous up to the boundary of $Y$. We cannot use $m=1$, for the function $z^{-1}$ is not in $L^{2}$ near the origin.

Proof. We can calculate $S\left(z^{-1 / m}\right)$ explicitly, and find that it is equal to -1 . Next we estimate the distance between $P(\varphi F)$ and $F$ in the Bergman space: by the Pythagorean theorem,

$$
\|P(\varphi F)-F\|^{2}=\|\varphi F-F\|^{2}-\|\varphi F-P(\varphi F)\|^{2} \leqslant\|\varphi F-F\|^{2} .
$$

Now $\|\varphi F-F\|=\|(1-\varphi) F\|$ can be made arbitrarily small if only $\varphi$ is suitably chosen. Lemma 6.3 shows that when $\|P(\varphi F)-F\|$ is small enough, the coefficient $h$ of $P(\varphi F)$ is in $N(Y)$, and $S(h)=S\left(z^{-1 / m}\right)=-1$.

We note that the coefficient $h$ of $P(\varphi F)$ satisfies $h(z, w)=h(z,|w|)$ in view of Proposition 3.1-or rather its analogue for $\mathcal{O}^{2}(Y)$. Now if $h$ were continuous up to the boundary, we would have $S(h) \in \mathbf{N}$ by Lemma 6.2 . This contradicts $S(h)=-1$ and proves the theorem. 


\section{References}

Barrett, David. 1984. Irregularity of the Bergman projection on a smooth bounded domain in $\mathbf{C}^{2}$. Ann. of Math. 119, 431-436.

Barrett, David. 1986. Biholomorphic domains with inequivalent boundaries. Inventiones Math. 85, 373-377.

Boas, Harold P. \& Straube, Emil J. 1989. Complete Hartogs domains in $\mathbf{C}^{2}$ have regular Bergman and Szegö projections. Math. Z. 201, 441-454.

Bonami, Aline \& Charpentier, Philippe. 1988. Une estimation Sobolev 1/2 pour le projecteur de Bergman. C. R. Acad. Sci. Paris 307, Série I, 173-176.

Diederich, Klas \& Fornæss, John Erik. 1977. Pseudoconvex domains: An example with nontrivial Nebenhülle. Math. Ann. 225, 275-292.

Kiselman, Christer O. 1978. The partial Legendre transformation for plurisubharmonic functions. Inventiones Math. 49, 137-148.

Krantz, Steven G. 1982. Function theory in several complex variables. John Wiley \& Sons. 